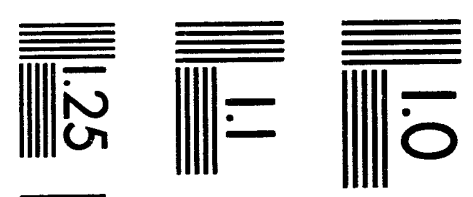

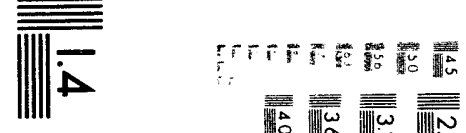

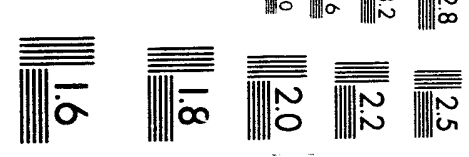



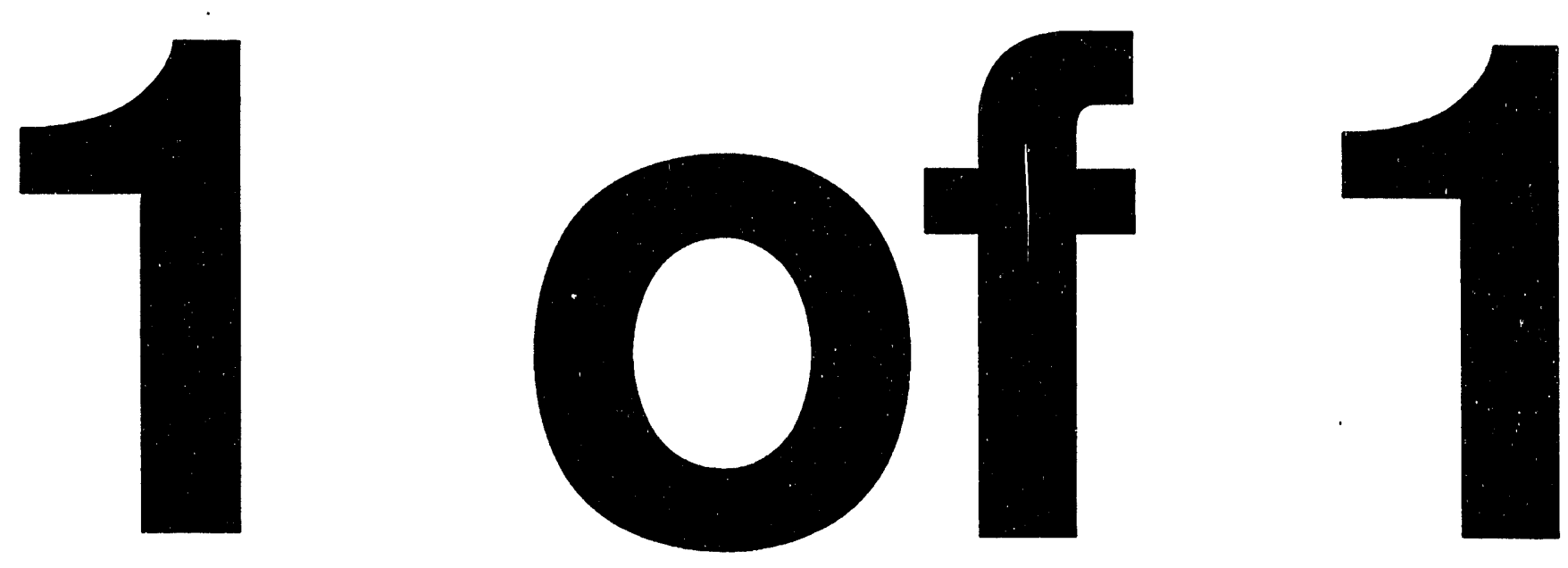


\title{
An Equal-Order Approximate Projection FEM
}

\author{
P.M. Gresho \\ S.T. Chan \\ M. Christon
}

This paper was prepared for submittal to the Tenth International Conference on Computational Methods in Water Resources Heidelberg, Germany July 19-23, 1994

February 1994

This is a preprint of a paper intended for publication in a journal or proceedings. Since changes may be made before publication, this preprint is made available with the understanding that it will not be cited or reproduced without the permission of the author.

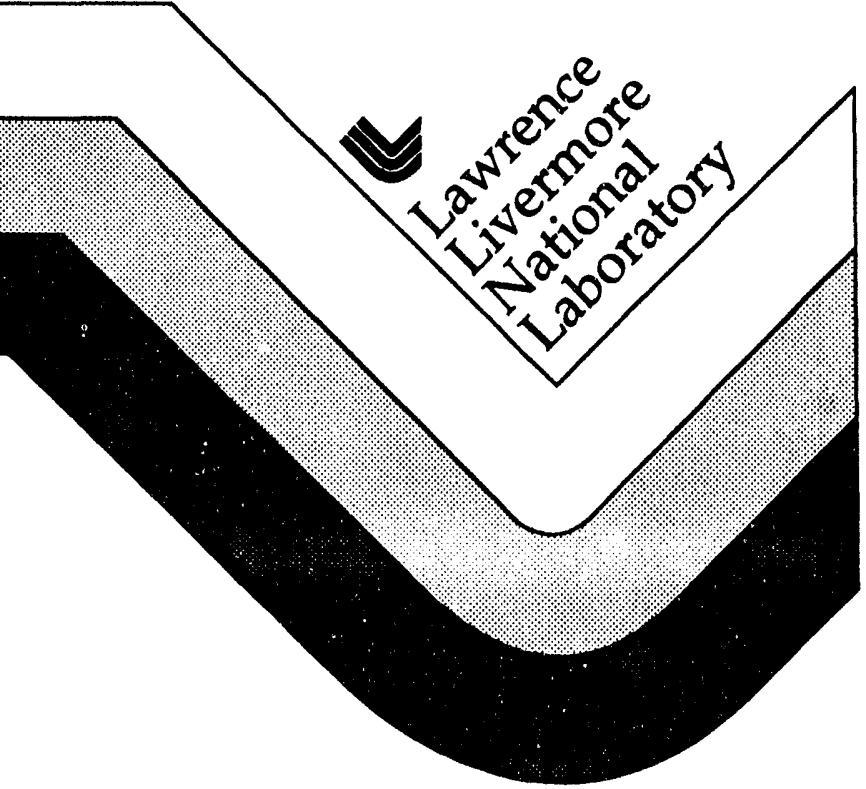




\section{DISCLAIMER}

This document was prepared as an account of work sponsored by an agency of the United States Government. Neither the United States Government nor the University of California nor any of their employees, makes any warranty, express or implied, or assumes any legal liability or responsibility for the accuracy, completeness, or usefulness of any information, apparatus, product, or process disclosed, or represents that its use would not infringe privately owned rights. Reference herein to any specific commercial products, process, or service by trade name, trademark, manufacturer, or otherwise, does not necessarily constitute or imply its endorsement, recommendation, or favoring by the United States Government or the University of California. The views and opinions of authors expressed herein do not necessarily state or reflect those of the United States Government or the University of California, and shall not be used for advertising or product endorsement purposes. 
AN EQUAL-ORDER APPROXIMATE PROJECTION FEM

P.M. GRESHO, S.T. CHAN, and M. CHRISTON

Physical Sciences Department

Lawrence Livermore National Laboratory

P.O. Box 808, L-262

Livermore, California 94551, USA

In this short paper we introduce and test a technique for solving the incompressible Navier-Stokes equations using bilinear basis functions for velocity and pressure.

\section{INTRODUCTION}

The equations of principal interest are

$\partial \underline{u} / \partial t+\underline{u} \cdot \nabla \underline{u}+\nabla P=v \nabla^{2} \underline{u}$ and $\nabla \cdot \underline{u}=0$ in $\Omega$,

with $\underline{u}=\underline{w}(t)$ on $\Gamma$ and $\int_{\Gamma} \underline{n} \cdot \underline{w}=0$,

and $\underline{u}=\underline{u}_{0}$ with $\nabla \cdot \underline{u}_{0}=0$ in $\Omega$ at $t=0$ and with $\underline{n} \cdot \underline{u}_{0}=\underline{n} \cdot \underline{w}(0)$ on $\Gamma$.

Since these "primitive equations" are usually considered as too difficult to solve as stated (fullycoupled), the following pressure Poisson equation (PPE) formulation is of much interest: Using $\partial(\nabla \cdot \underline{u}) / \partial t=0$ in (1) leads to the PPE:

$\nabla^{2} P=\nabla \cdot\left(\nu \nabla^{2} \underline{u}-\underline{u} \cdot \nabla \underline{u}\right) \equiv \nabla \cdot \underline{a}$ in $\Omega, \partial P / \partial n=\underline{n} \cdot\left(v \nabla^{2} \underline{u}-\underline{u} \cdot \nabla \underline{u}-\frac{\partial \underline{w}}{\partial t}\right)$ on $\Gamma .(5),(6)$

The PPE (derived) formulation is (1) and (3)-(6): i.e., (2) is omitted because it is implied [See, e.g., Gresho (1991a)].

The weak (Galerkin) form of the PPE formulation is

$$
\begin{aligned}
& \int \underline{\mathrm{v}} \cdot\left(\frac{\partial \underline{\underline{u}}}{\partial t}+\underline{\underline{u}} \cdot \nabla \underline{\underline{u}}\right)+v(\nabla \underline{u})^{T}: \nabla \underline{\underline{v}}-P \nabla \cdot \underline{\mathrm{v}}=0 \quad \forall \underline{\mathrm{v}} \in \underline{H}_{O}^{1} \\
& \text { and } \int \nabla \varphi \cdot \nabla P=\int \underline{a} \cdot \nabla \varphi-\int_{\Gamma} \varphi \underline{\underline{n}} \cdot \partial \underline{w} / \partial t \quad \forall \varphi \in H_{0}^{l} .
\end{aligned}
$$

\section{Remarks:}

1. The above PPE formulation assures that $\nabla \cdot \underline{u}=0$ in a very weak (and vague) sense in that if (7) and $(8) \Rightarrow(1)$ and (3)-(6) then $\nabla \cdot \underline{u}=0$. I.e., if our weak solution is also a classical solution, then $\underline{u}$ will be (strongly) div-free. These are big if's. 
2. The PPE formulation has more solutions than the $\underline{u}-P$ formulation-and the extras are spurious, a particular example being that $\nabla \cdot \underline{u}_{0}$ need not vanish in order that PPE solutions exist-any $\underline{u}_{0}$ 'works'. [See Gresho (1992) for others.]

If we discretize (1)-(4) via the GFEM, we arrive at the following Index 2 DAE system:

$$
M \dot{u}+N(u) u+C P=K u+f, u(0)=u_{0} \text { and } C^{T} u=g \text {, with } C^{T} u_{0}=g_{0}
$$

where $f$ and $g$ correspond to $\mathrm{BC}$ data. Note that (9) also corresponds to the finite-dimensional version of (7). Converting this to a lower index (easier) problem is achieved by inserting $\dot{u}$ into the time-differentiated version of (10): the resulting Index 1 DAE system is (9) and

$$
\left(C^{T} M^{-1} C\right) P=C^{T} M^{-1}(K u+f-N(u) u)-\dot{g} \text {. }
$$

As with the PPE formulation, the Index 1 formulation uncouples the pressure from the acceleration (its "raison d" etre"). Also as with the continuous formulation, violation of $C$ " $u_{0}=g_{0}$ results in an ill-posed Index 2 problem but the Index 1 version-having more solutions-is not ill-posed. It is just not right; the resulting discrete velocity field will not satisfy $C^{T} u=g$. [It will satisfy $C^{T} u=g+C^{T} u_{0}-g_{0} ;$ see Gresho (1991b).].

For comparison with what follows below, we now introduce the projection matrix, 8 :

$$
\wp \equiv I-M^{-1} C\left(C^{T} M^{-1} C\right)^{-1} C^{T} \text {, }
$$

which is a 'formal' construction only. $\wp$ has the following properties:

1. $\wp^{2}=\wp$; it satisfies the definition of a projection.

2. If $w$ is an arbitrary velocity field, then $\wp w$ is its projection to the (discretely) div-free subspace; i.e. $u \equiv \wp w$ satisfies $C^{T} u=0$. In fact, $C^{T} \wp \equiv 0$.

3. The eigenvalues of $\wp$ are either zero or 1 , and its norm is unity. All discretely divergence-free vector fields are eigenvectors of $\wp$ with eigenvalue unity.

In terms of $\wp$, the Index 1 problem can be 'condensed' to an Index 0 problem (i.e., to a set of ODE's):

$$
\dot{u}=\wp\left[M^{-1}(K u+f-N(u) u)\right]+M^{-1} C\left(C^{T} M^{-1} C\right)^{-1} \dot{\boldsymbol{g}} \text {, }
$$

which clearly satisfies $C^{T} \dot{u}=\dot{g}$; (only) an initialiy div-free velocity field will remain div-free.

Suppose though that we use instead (7) and (8) to generate our GFEM equations? The result is (9) and

$$
L P=h, \text { where } L_{i j} \approx \int \nabla \varphi_{i} \cdot \nabla \varphi_{j}
$$

is the 'conventional' GFEM Laplacian and $\varphi_{k}$ is the bilinear basis function associated with node $k$. The RHS vector is

$$
h_{i} \equiv \int \nabla \varphi_{i} \cdot\left(v \nabla^{2} \underline{u}^{h}-\underline{u}^{h} \cdot \nabla \underline{u}^{h}\right)-\int_{\Gamma} \varphi_{i} \underline{n} \cdot \partial \underline{w} / \partial t
$$

from the finite-dimensional form of (8). Clearly some remedial action is required if $C^{0}$ basis functions are to be employed (for which $\nabla^{2} \underline{u}^{h}$ is not well-defined); for one type of response, see Sohn and Heinrich (1990). For a 'rationalization', note that $v\left|\nabla \varphi \cdot \nabla^{2} \underline{\mu}=v\right| \underline{\varphi n} \cdot \nabla^{2} \underline{u}$ when $\nabla \cdot \underline{u}=0$ and thus the omission of the viscous term in (16) can only 'affect things' near $\Gamma$-an especially valid approximation for large Reynolds number (small $v$ ). (See Hassanzadeh $e t$ al. 1994) In our formulation, an approximation to (14)-(16) is actually only required at $t=0-10$ estimate the initial pressure field; for $t>0$, the pressure is determined by the (approximate) projection method-described below. 
We, and many others, have generated codes based on the Index 1 formulation of (9) and (11) using the $Q_{1} P_{0}$ element; i.e., the quadrilateral element with bilinear basis functions (2D version) for velocity and piecewise-censtant basis functions for $P$. But, with the exception of our ad hoc procedure in Gresho and Chan (1990), all $Q_{1} P_{0}$ Index 1 codes 'required' the also ad hoc (and more deleterious) approximation of mass lumping (because $M^{-1}$ is otherwise dense), which introduces a serious loss of accuracy for the bilinear element when the flow is advection-dominated (vortex shedding, for example). Additional bad features of this element are:

1. It suffers from the "Bent-Element Blues"- (see Gresho and Leone , 1984); the $C P$ approximation to $\nabla P$ is not very accurate when the elements are bent pretty hard (are far from rectangular).

2. The staggered mesh 'bookkeeping' is not fun.

3. The Laplacian matrix $C^{T} M_{L}^{-1} C$ is 'awkward' and is suspected to converge 'too slowly' when iterative solvers are used. ( $M_{L}$ is the lumped version of $M$.)

4. It fails the LBB test! I.e., the velocity and pressure spaces are not quite compatible.

5. The (necessary) integration by parts of the $\nabla P$ term causes 'problems' in 'flow-through' domains wherein a homogeneous normal natural boundary condition $\left(v \partial u_{n} / \partial n-P=0\right)$ is employed and a significant 'body force' is present [see Sani and Gresho (1994)].

\section{THEORY}

So, to get back 'in vogue', we now describe our attempt at creating a stabilized equal-order element; viz $Q_{1} Q_{1}$, which overcomes at least flaws (1), (2), and (5) above. But (3) and (4) still 'shoot it down' and mass lumping still seems required. To implement a consistent mass $Q_{1} Q_{1}$ element and to pass 'LBB' (which $Q_{1} Q_{1}$ fails in spades), we invoke an "approximate projection" [Almgren $e t$ al. (1993), and Dvinsky and Dukowicz (1993)] in much the same way that many before us have done, viz, we replace the 'bad' Laplacian in (11) by the good (conventional GFEM) Laplacian, (15). Actually, the procedure is closer to the following:

1. Replace $-P \nabla \cdot \underline{v}$ in (7) by $\underline{v} \cdot \nabla P$

which implies $v \partial \underline{u} / \partial n=\underline{0}$ as an $\mathrm{OBC}$ which is generally an ill-posed boundary condition (see Sani and Gresho 1994). But we get away with this crime because we will no longer require a 'stringent' version (even discretely) of $\nabla \cdot \underline{u}=0$.

2. Generate the following Index 2 DAE's:

$$
M \dot{u}+N(u) u+G P=K u+f, u(0)=u_{0} \text { and } D u=8, D u_{0}=80
$$

where no longer are $G$ and $D$ transposes of each other.

3. Solve the DAE's via the following approximate projection method: Given $u_{n}$ and $P_{n}$,

(i) Solve $M \frac{\left(\tilde{u}_{n+1}-u_{n}\right)}{\Delta t}+N\left(u_{n}\right) u_{n}+G P_{n}=\frac{1}{2} K\left(\tilde{u}_{n+1}+u_{n}\right)+f_{n}$ for $\tilde{u}_{n+1}$.

(ii) Project $\tilde{u}_{n+1}$ to the approximately discretely div-free subspace as follows:

(1) Solve $\Delta t L\left(P_{n+1}-P_{n}\right)=D\left(\tilde{u}_{n+1}-u_{n}\right)-\left(g_{n+1}-g_{n}\right)$ for $\Delta P$.

(2) Compute the projected velocity from $u_{n+1}=\tilde{u}_{n+1}-\Delta t M_{L}^{-1} G\left(P_{n+1}-P_{n}\right)$

(3) Update $P$ and go to (i)

The approximate projection above is derived as follows: Given $\tilde{u}_{n+1}$, solve

$$
M_{L} \frac{\left(u_{n+1}-\tilde{u}_{n+1}\right)}{\Delta t}+G\left(P_{n+1}-P_{n}\right)=0 \text { and } D u_{n+1}=g_{n+1}+\left(D u_{n}-g_{n}\right) \text {, }
$$


which is called 'projecting the difference' - a required 'trick' obtained from J. Bell (LLNL, personal communication). Note that, because of the next trick, below, $D u=g$ is never quite achieved in this method.

These equations imply the following 'PPE' for the pressure update:

$$
\Delta t\left(D M_{L}^{-1} G\right)\left(P_{n+1}-P_{n}\right)=D\left(\tilde{u}_{n+1}-u_{n}\right)-\left(g_{n+1}-g_{n}\right) \text {. }
$$

which is replaced by (22) $\left(D M_{L}^{-1} G \rightarrow L\right)$ and is the final "trick" referred to above-a replacement made necessary because $D M_{L}^{1} G$ has too many spurious pressure modes (fails LBB) and made 'interesting' because iterative solvers should like $L$ better (i.e., require fewer iterations).

Remarks:

If $D u_{n}-g_{n}$ is omitted from the RHS of (22), the results are disappointing in that (at least) a steady pressure cannot be attained even when the velocity becomes steady.

Now comes the 'hard part' - analyzing the resulting algorithm to show that it is actually useful. And we admit up front that we have not yet been totally successful. But, being more like engineers than mathematicians (mathematical engineers?), we tested it in the computational laboratory anyway. (And it works. This happens often with engineers - but definitely not always.) If we study (21), (22), and (23) for $\Delta t \rightarrow 0$ we get, with $F_{n} \equiv f_{n}+K u_{n}-N\left(u_{n}\right) u_{n}-G P_{n}$,

(i) $\tilde{u}_{n+1}=u_{n}+\Delta t M^{-1} F_{n}+O\left(\Delta t^{2}\right)$

(ii) $L\left(P_{n+1}-P_{n}\right)=D M^{-1} F_{n}-\dot{g}_{n}+O(\Delta t)$

(iii) $u_{n+1}=\tilde{u}_{n+1}-\Delta t M_{L}^{-1} G L^{-1}\left\{D M^{-1} F_{n}-\dot{g}_{n}\right\}+O\left(\Delta t^{2}\right)$

$$
\equiv u_{n}+\Delta t\left[\wp_{a} M^{-1} F_{n}+M_{L}^{-1} G L^{-1} \dot{g}_{n}\right]+O\left(\Delta t^{2}\right)
$$

(iv) $I u_{n+1}=D u_{n}+\Delta t\left[E L\left(P_{n+1}-P_{n}\right)+\dot{g}_{n}\right]+O\left(\Delta t^{2}\right)$

where $E \equiv I-D M_{L}^{-1} G L^{-1}$ and $E^{n} D=D \wp_{a}^{n}$

(v) Thus $\Delta t \rightarrow 0 \Rightarrow \dot{u}=\wp_{a} M^{-1}[f+K u-N(u) u-G P]+M_{L}^{-1} G L^{-1} \dot{g}$

(vi) $\quad D \dot{u}=D \wp_{a} M^{-1} F+D M_{L}^{-1} G L^{-1} \dot{g}=E D M^{-1}[f+K u-N(u) u-G P]+\left(D M_{L}^{-1} G\right) L^{-1} \dot{g}$ and the problem is to reconcile (iv) with (vi); the former $\Rightarrow D \dot{u}=E I . \cdot \lim \left(P_{n+1}-P_{n}\right)+\dot{g}$ and the two are only in accord if $P_{n+1}-P_{n} \rightarrow 0$ as $\Delta t \rightarrow 0$, and if $\Delta t \rightarrow 0$

$$
\left(D M^{-1} G\right) P=D M{ }^{-1}[f+K u-N(u) u]-\dot{\boldsymbol{g}} \text {. }
$$

which is just the PPE that we' $d$ like the pressure to obey! And if this is true, then $(v)$ above yields $\dot{u}=M^{-1}[f+K u-G P-N(u) u]$,

also the desired result. (Note the mysterious disappearance of the $L$-matrix.) But-we have not been able to show that $P_{n+1}-P_{n}=O(\Delta t)$. In fact, somewhat the converse seems to be true; viz, starting from the initial pressure, $P_{0}$, obtained from

$$
L P_{0}=D M^{-1}\left[f_{0}+K u_{0}-N\left(u_{0}\right) u_{0}\right]-\dot{g}_{0} \text {, }
$$

proceeding through a few steps, and generalizing via induction leads to

$$
\begin{aligned}
& L\left(P_{n+1}-P_{n}\right)=E^{n+1} L P_{0}+O(\Delta t) \text { and to } \\
& D u_{n+1}=D u_{n}+\Delta t\left[E^{n+2} L P_{0}+\dot{g}_{n}\right]+O\left(\Delta t^{2}\right) .
\end{aligned}
$$


Thus, at least initially, $P_{n+1}-P_{n}=O(1)$ in $\Delta t$ and, for large $n$, we recover to $P_{n+1}-P_{n}=O(\Delta t)$ iff ${ }^{n+1} \rightarrow 0$. But since that $E$ has eigenvectors with unit eigenvalues whenever the initial vector has any non-zero projection onto the null space of $G, E^{n}$ may not $\rightarrow 0$. It seems that only if $L P_{0}$ (and $\therefore P_{0}$ ) has no spurious pressure node components can we assert that the algorithm can 'recover' - and even then the early (small $n$ ) results are likely to be poor...

But the resulting code seems to perform much better than these gloomy results would indicate-as we shall show.

\section{NUMERICAL RESULTS}

We have successfully (for the most part) compared $Q_{1} Q_{1}$ against our workhorse $Q_{1} P_{0}$ code for liddriven cavities and vortex sheddng past circular cylinders. Here we show a sample of results for flow past an airfoil at $\operatorname{Re}=10^{4}$ (based on chord, $c$ ) at a small $\left(1.2^{\circ}\right)$ angle of attack. The particular airfoil, "a NACA 16 thickness form with maximum thickness $t_{0} / c=8.84 \%$ and maximum camber $2.576 \%$ with a beveled anti-singing trailing edge"-E. H. Lurie (1993), was tested in a water tunnel (incompressible flow!) at MIT's Ocean Engineering Department as part of a Navy/ARPA program on unsteady fluid dynamics. But our laminar flow simulations were not meant to be compared with their measured data at $\operatorname{Re}>10^{6}$ because we do not yet have a believable turbulence model. Maybe later.

We designed a 'truth' mesh of $-40,000$ elements and a test mesh of -6400 elements. The fine mesh was run at $\operatorname{Re}=10^{3}, 10^{4}, 10^{5}$, and $10^{6}$ using $Q_{1} P_{0}$ and our 'projection 2' algorithm (Gresho and Chan 1990). The results were not believable at $\operatorname{Re}=10^{6}$ (chaotic), semi-so at $\operatorname{Re}=10^{5}$ (nearly periodic), and believable at $\operatorname{Re} \leq 10^{4} . \operatorname{Re}=10^{3}$ (only) produced a steady-state result. The coarse mesh for $\operatorname{Re}=10^{3}$ was then run with $Q_{1} P_{0}$ and the new $Q_{1} Q_{1}$; all three results were acceptably close to each other.

We now focus on the $\operatorname{Re}=10^{4}$ case and compare $Q_{1} P_{0}$ on the two meshes with $Q_{1} Q_{1}$ on the coarse mesh. Qualitatively, the flow is one of periodic vortex formation and shedding of vortices at the trailing edge. Nowhere else is there any interesting dynamics. Fig. 1 shows snapshots of vectors, streamfunction, and vorticity from the coarse mesh (vectors are interpolated via a coarseryet mesh graphics package.). Fig. 2 shows time histories, starting from potential flow, of the $x$ component of velocity at a node just above $(\sim .002 c)$ the trailing edge-and the pressure there, as well as the RMS-norm of the discrete divergence for $Q_{1} Q_{1}$. The agreement of $Q_{1} Q_{1}$ with $Q_{1} P_{0}$ on the coarse mesh is quite close - the range of $u^{\prime} s$ during vortex shedding being $\sim-.07$ to +.16 for $Q_{1} P_{0}(2 c)$ and --.08 to +.15 for $Q_{1} Q_{1}(2 e)$. (The fine mesh results (2a) show $\sim-.10$ to +.20 , somewhat stronger, but still reasonably close). The corresponding pressure histories are (Dank Gott!) of a similar quality; the coarse mesh results ranged from $\sim-.17$ to -.31 for $Q_{1} P_{0}(2 \mathrm{~d})$ and $\sim-.16$ to -.27 for $Q_{1} Q_{1}$ (2f), compared to the 'true' results $(2 \mathrm{~b})$ of $\sim-.15$ to -.33 . (In all cases, the inlet velocity is 1.0 , as is that on the top and bottom boundaries (tow tank BC's). The OBC's were: 'natural'; i.e., $v \partial u / \partial x-P=0=v \partial v / \partial x$ for $Q_{1} P_{0}$ and $v \partial u / \partial x=v \partial v / \partial y=P=0$ for $Q_{1} Q_{1}$, the latter being imposed as an essential $\mathrm{BC}$ in the PPE.). 


\section{CONCLUSIONS}

While interesting and viable (apparently -in spite of the shortcomings in our analysis), the new $Q_{1} Q_{1}$ has not yet displaced 'old faithful' ( $\left.Q_{1} P_{0}\right)$, largely because the PPE solution-via DSCGwas little cheaper (10-15\% fewer iterations). Perhaps it would look better using multi-grid.

For a simpler application of this $Q_{1} Q_{1}$ stabilization technique using forward Euler time marching, see Gresho and Chan (1994).

Work performed under the auspices of the U.S. Department of Energy by the Lawrence Livermore National Laboratory under contract No. W-7405-Eng-48.

\section{REFERENCES}

Gresho, P.M., and Leone, J. (1984) "Another attempt to overcome the bent element blues," 5th Int. Conf. on FEM in Proceedings, Water Resources; Burlington VT, Springer-Verlag, pp. $667-683$.

Gresho, P.M. (1991a) "Incompressible fluid dynamics: Some fundamental formulation issues", Annu. Rev. Fluid Mech., Vol. 23, pp. 413-453

Gresho, P.M. (1992) "Some interesting issues in incompressible fluid dynamics, both in the continuum and in numerical simulation", Advances in Applied Mechanics, Vol. 28, pp. 46-133.

Gresho, P.M. (1991b) "Some current CFD issues relevant to the incompressible Navier-Stokes equations", Computer Methods in Applied Mechanics and Engineering, Vol. 87, pp. 201-252.

Sohn, J.L., and Heinrich, J.C. (1990) "A poisson equation formulation for pressure calculations in penalty finite element models for viscous incompressible flows", Int. J. Num. Meth. Fluids, Vol. 30, pp. 349-361.

Hassanzadeh, S., Sonnad, V., and Foresti, S. (1993) "Finite element implementation of boundary conditions for the pressure poisson equation of incompressible flow", to appear in Int. J. Num. Meth. Fluids (1994).

Gresho, P.M., and Chan, S.T. (1993) "On the theory of semi-implicit projection methods for viscous incompressible flow and its implementation via a finite element method that also introduces a nearly consistent mass matrix. Part 2: Implementation", Int. J. Num. Meth. Fluids, Vol. 11, pp. 621-659.

Sani, R.L., and Gresho, P.M. (1993) "Resume and remarks on the spen boundary condition minisymposium", to appear in Int. J. Num. Meth. Fluids.

Almgren, A.S., Bell, J.B., and Szymczak, W.G. (1993) "A numerical method for the incompressible Navier-Stokes equations based on an approximate projection", submitted to SLAM J. of Sci. Comp.

Dvinsky, A.S., and Dukowicz, J.K. (1992) "Null-space-free methods for the incompressible Navier-Stokes equations on non-staggered curvilinear grids", Computers Fluids, Vol. 22. pp. 685-696.

Lurie, E.H. (1993) "Unsteady response of a two-dimensional hydrofoil subject to high reduced frequency gust loading", Ocean Engineering's Report No. 93-5.

Gresho, P.M., and Chan, S.T. (1994) “A Another stabilized incompressible Navier-Stokes solver using explicit time-marching", in Proceedings, Second US-Japan Symposium on Finite Element Methods in Large-Scale CFD, Chuo University, 14-16 March 1994. 
(a)

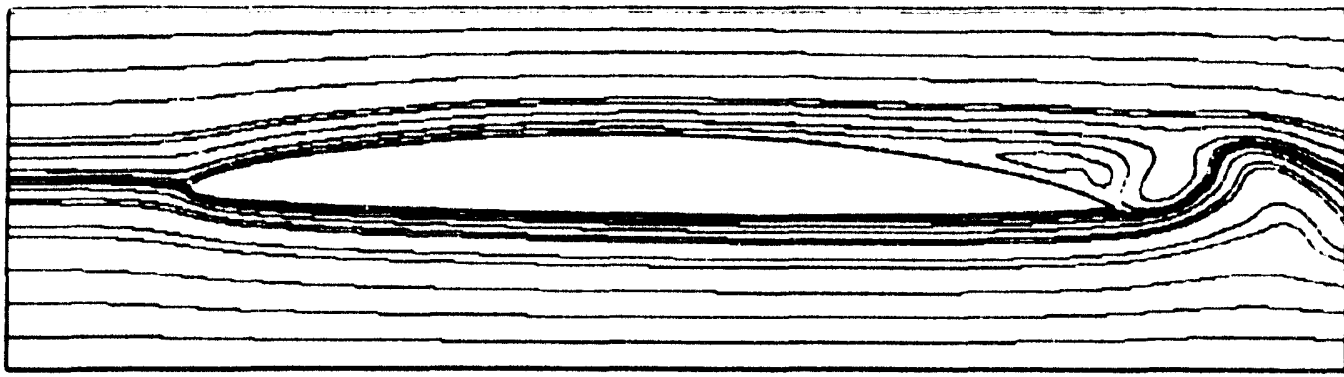

(b)

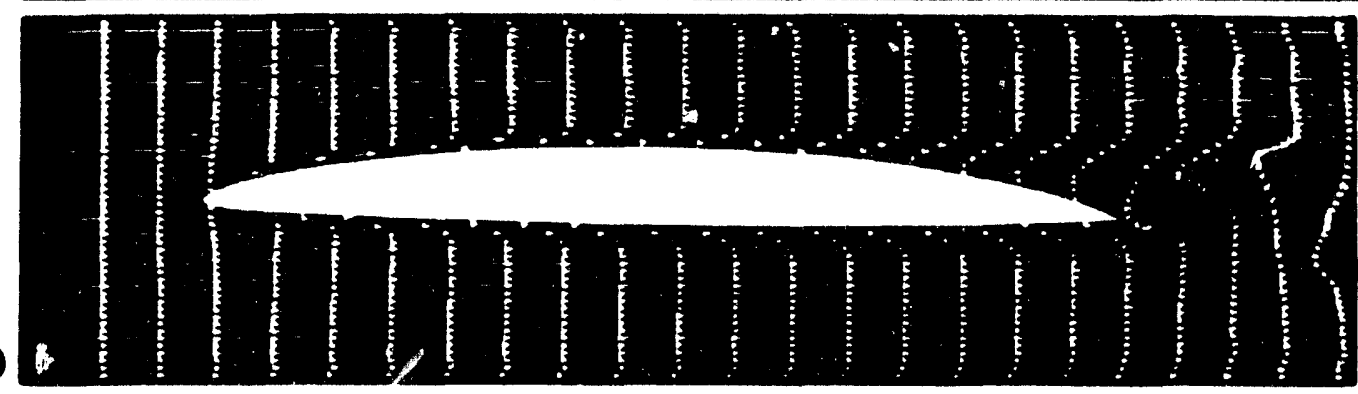

(c)
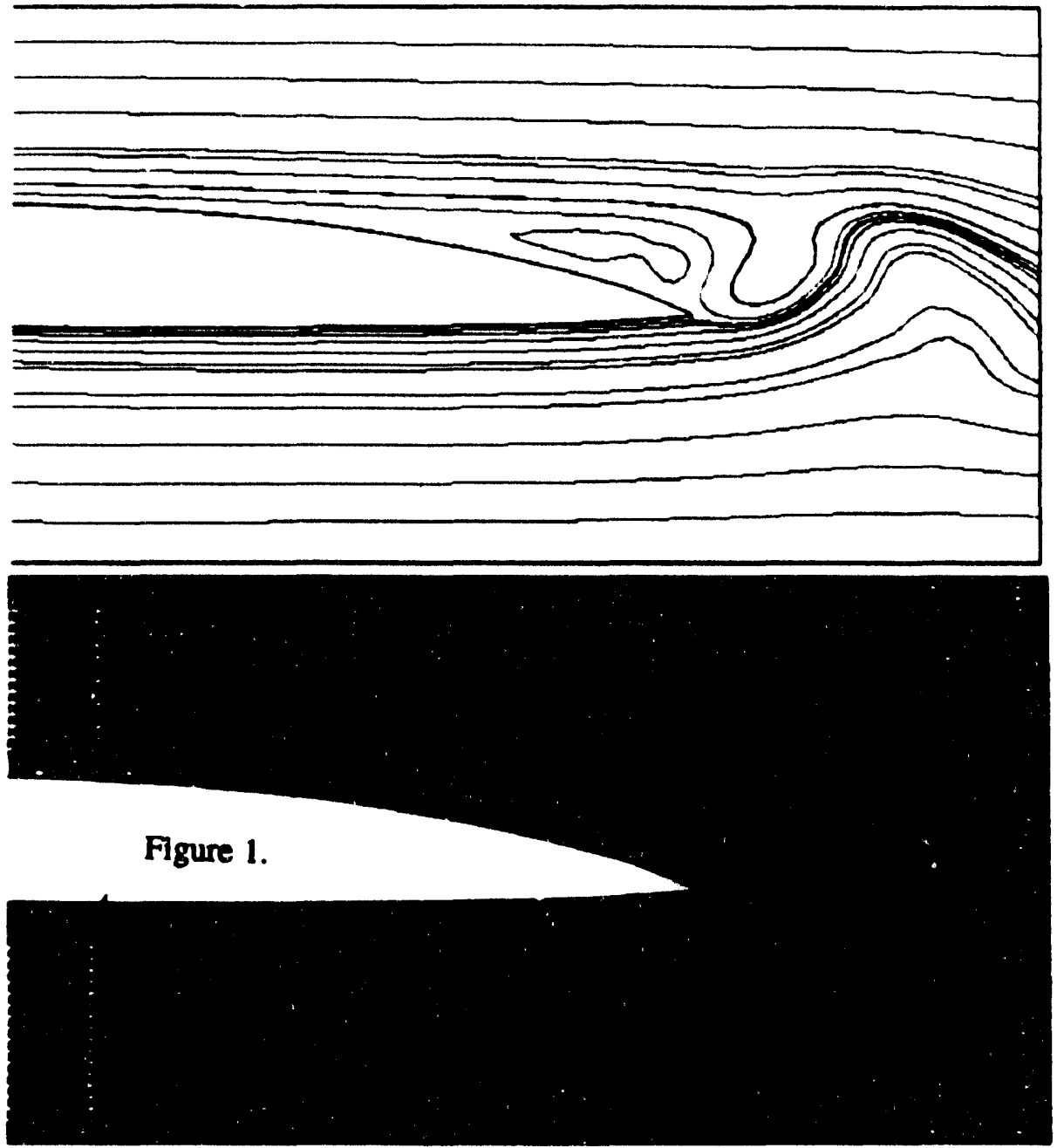

(d) 

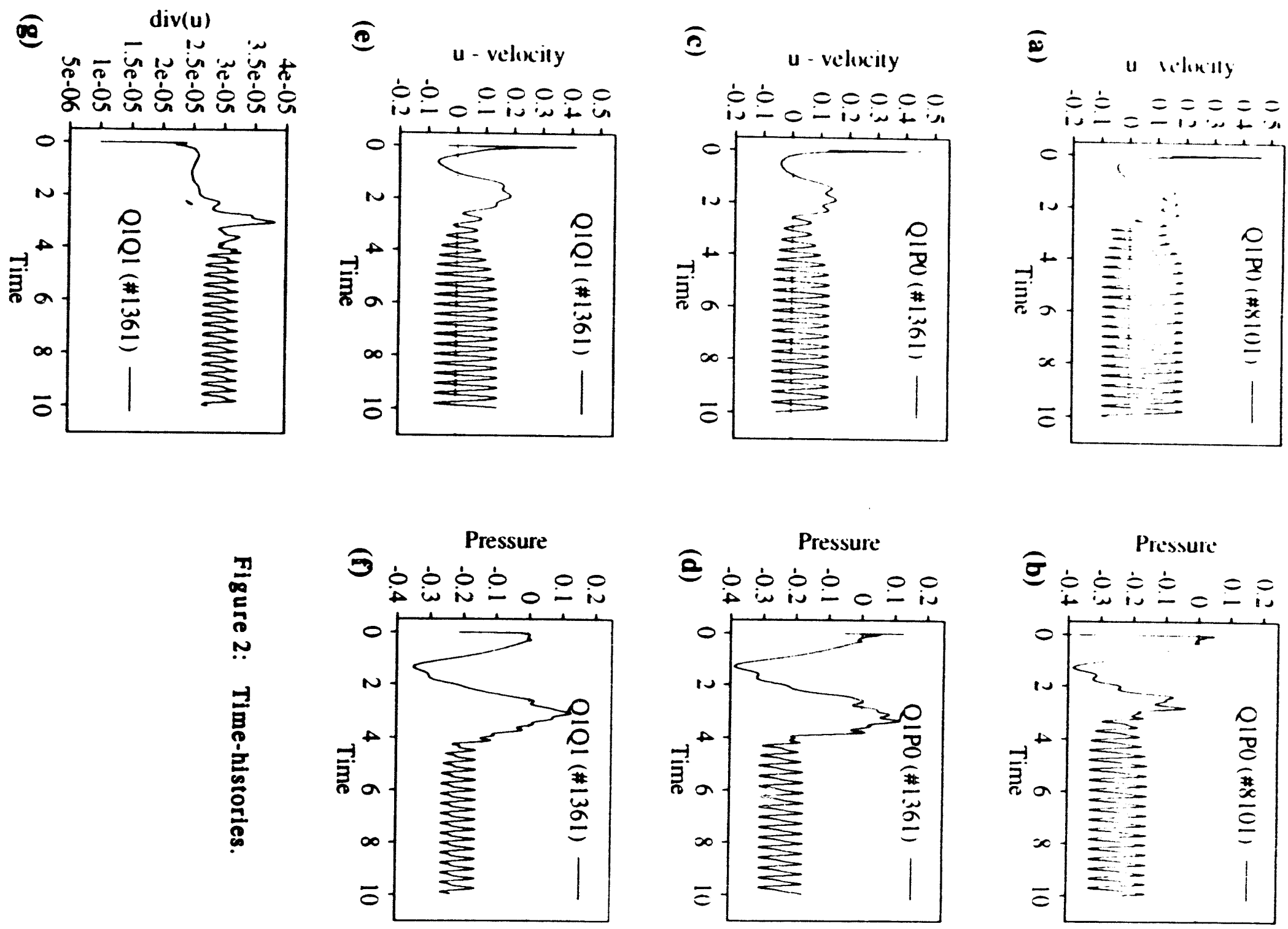

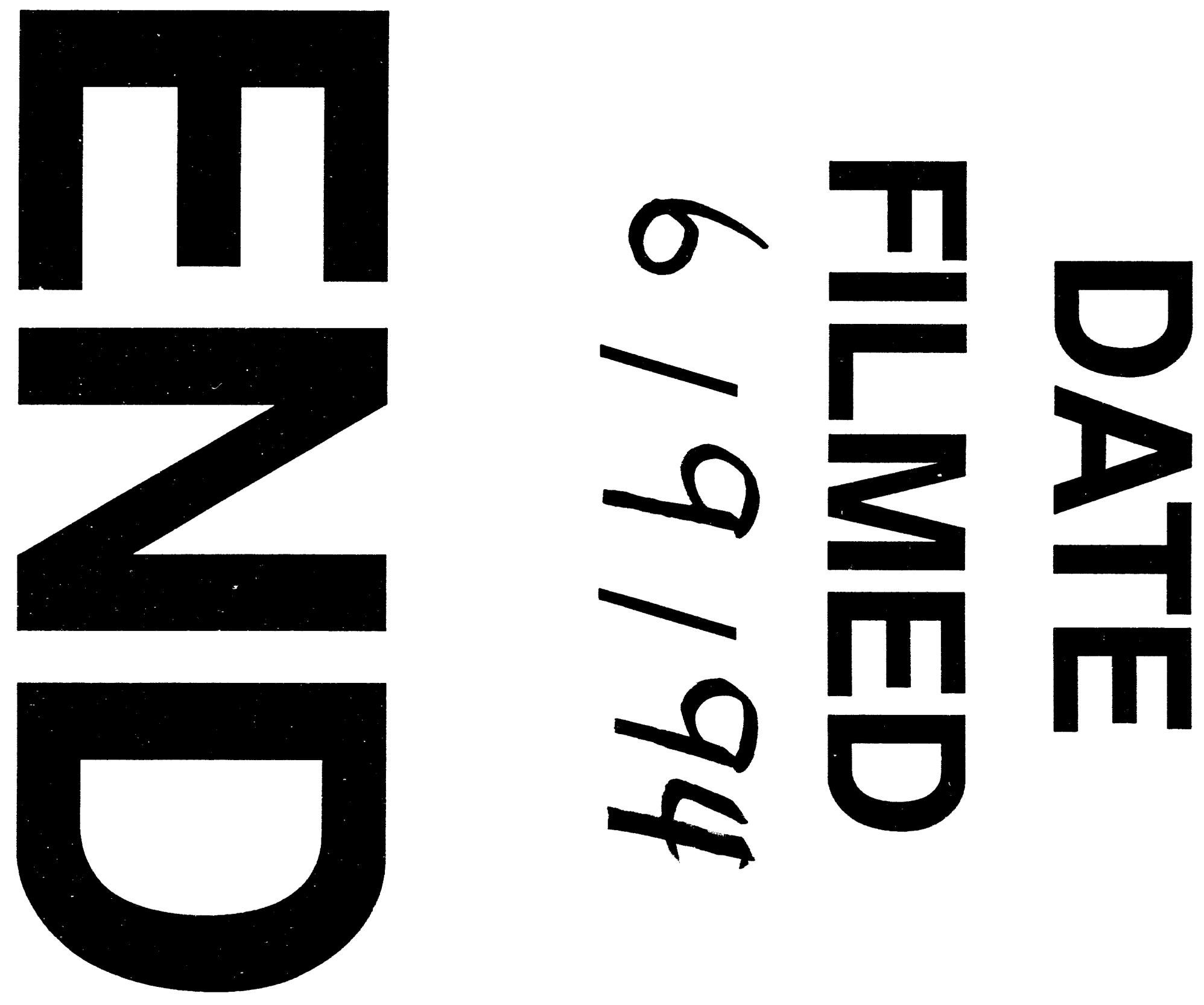